\section{Kraft für neue Ideen}

_ Das neue Jahr ist gerade einmal acht Tage jung. Wie sieht es mit Ihren guten Vorsätzen aus? Haben Sie bereits die Ärmel hochgekrempelt, um diese umzusetzen? Haben Sie sie mittlerweile längst wieder über Bord geworfen? Oder halten Sie es wie die Japaner und planen eine sogenannte Bonenkai - eine Feier zum Vergessen des zurückliegenden Jahres? Im Durchschnitt nimmt ein Japaner am Jahresende an 2,6 solcher Veranstaltungen teil und zieht dort mit Kollegen oder Freunden Bilanz.

_ Resümieren und reflektieren. Diese Tradition kann man sich zum Vorbild nehmen, um Vergangenes abzuschließen und dabei Kraft für neue Ideen zu sammeln. Nicht immer aber sind neue Projekte schnell abgeschlossen, sondern ziehen sich auch einmal über einen längeren Zeitraum. So wie etwa das der Ergotherapeutin Annika Grote in der Gemeinde Flein. Sie stampfte dort zusammen mit dem Bürgermeister und zahlreichen Engagierten verschiedene Angebote zur Gesundheitsförderung und Prävention aus dem Boden und berichtet darüber ab Seite 8.

_ Ein weiteres Projekt fand im letzten Jahr an einer Ergotherapieschule in Leipzig statt. Dort beschäftigte sich eine Klasse im Fach Medizinsoziologie mit dem Thema Sterben. Daraus entstanden ist ein Artikel für ergopraxis, den sie ab Seite 24 lesen können.

_ Vielleicht arbeiten Sie in einer Praxis und haben sich im vergangenen Jahr regelmäßig darüber geärgert, dass der fachliche Austausch mit den verordnenden Ärzten einfach nicht klappen will? Dann erfahren Sie ab Seite 30, woran das liegen könnte und wie Sie die Lage in Zukunft verbessern können.

_ Pünktlich zum Jahresbeginn titelt unsere Beilage praxisprofi mit „Mehr als nur gute Vorsätze“. Sie erhalten zum Beispiel Anregungen, wie Sie als Praxisinhaber oder -inhaberin Mitarbeitergespräche führen können oder nach welchen Kriterien Sie Ihre Angebotspalette erweitern können.

Ich wünsche Ihnen fürs neue Jahr eine große Portion Energie, Ihre persönlichen Projekte erfolgreich in die Tat umzusetzen. Damit Sie am Ende des Jahres bei der „Feier zum Vergessen“ eine positive Schlussbilanz ziehen können!

Ihre

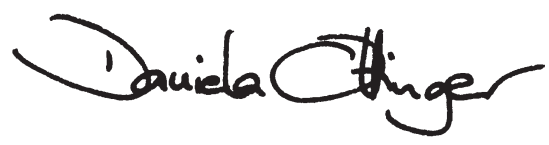

ZU GEWINNEN

Kursplatz

1 Kurs „Constraint-induced Movement Therapy“

Seite 43

Bücher

4-mal „Fallbuch Ergotherapie in

der Pädiatrie“

Seite 18

3-mal „Sterben, Tod

und Trauer"

Seite 26

3-mal „Gesprächstechniken -

Trainer"

Seite 31

Und außerdem...

2 Stoppuhren

Seite 35 